They were precipitated by strenuous exercise such as bicycle riding and racing and running. He would continue to ride automatically during seizures. Neurologic exam and MRI were normal. Interictal EEG showed left temporal lobe epileptiform discharges. He continues to have seizures during exercise despite treatment with carbamazepine and gabapentin. Neither patient had seizures induced by hyperventilation, passive and active stationary limb movement or by imagining competitive sports. (Sturm JW, Fedi M, Berkovic SF, Reutens DC. Exercise-induced temporal lobe epilepsy. Neurology 2002;59:1246-1248). (Reprints: Dr David C Reutens, Department of Neurology, Austin and Repatriation Medical Centre, Studley Road, Heidelberg, Victoria 3084, Australia).

COMMENT. Reflex epilepsies in response to a well-defined precipitating stimulus occur in about $5 \%$ of patients with epilepsy. Exercise-induced epilepsies are usually generalized or frontal lobe in origin, and less commonly involve the temporal lobe. Other reflex precipitants for temporal lobe seizures include music, eating, hot water immersion, laughter, and thinking. The stimuli may involve emotional and motivational factors, and require complex processing in limbic structures. Exercise-induced seizures are rare and should not lead to a sedentary life-style. In fact, moderate exercise may offer some protection or improved control of epilepsy.

\title{
LANGUAGE DISORDER WITH FOCAL EPILEPSIES
}

The relationship between language disorder and epileptic seizures was examined in 109 children, ages 5-17 years, attending a national center for epilepsy over a 4 year period and at the University of Manchester, UK. Median age at onset of epilepsy was 2 years 5 months, and seizure onset was before 6 years of age in $89 \%$ of the cohort. Of the $46(42 \%)$ children with language disorders in the research sample, 30 had localization-related epilepsies and an additional 3 were unclassified. Less than $18 \%$ had generalized seizures. Twelve had Lennox-Gastaut syndrome, 7 temporal lobe epilepsy, 5 frontal lobe epilepsy, and 3 had a history of West syndrome. Children with focal epilepsies were $30 \%$ more likely to have language disorder than other language disability subtypes. Children with simple or complex partial seizures had an increased risk of language disorder. A routine screening test to check for language impairment is recommended in children with focal epilepsies. Early detection and therapy may prevent the development of cognitive, emotional, and behavior problems. (Parkinson GM. High incidence of language disorder in children with focal epilepsies. Dev Med Child Neurol 2002;44:533-537). (Respond: Dr Gillian M Parkinson, The David Lewis Centre for Epilepsy, Cheshire and the raculty of Education, University of Manchester, UK).

COMMENT. In addition to specific epileptic aphasias (Landau-Kleffner syndrome), language disorders occur with increased incidence in children with focal epilepsies. In the management of children with focal simple or complex partial seizures, an evaluation of language development is important. One in 5 children with language impairment have seizures (Robinson 1991), compared to a $5-7 \%$ seizure prevalence in the general childhood population. Reasons postulated for this association include altered brain function due to seizures, a genetic association, and a link between antecedent brain abnormalities and seizures leading to cerebral dysfunction in language centers.

Developmental language disorder associated with varying degrees of polymicrogyria is discussed in Ped Neur Briefs August 2002;16:58. An MRI should be considered in children with language disorder and focal seizures. 